\title{
Analyzing layered soil problems with an alternative multi-region BEM technique and a new infinite boundary element formulation
}

\author{
D. B. Ribeiro \& J. B. Paiva \\ Structural Engineering Department, São Carlos Engineering School, \\ University of São Paulo, Brazil
}

\begin{abstract}
The main objective of this work is to obtain an efficient three-dimensional boundary element (BE) formulation for layered soil simulation. This formulation is obtained by combining an alternative multi-region technique with an infinite boundary element (IBE) formulation. Kelvin fundamental solutions are employed, considering the static analysis of isotropic and linear-elastic domains.

Establishing relations between the displacement fundamental solutions of the different domains, the alternative technique used in this paper allows analyzing all domains as a single solid, not requiring equilibrium or compatibility equations. This approach also leads to a smaller system of equations when compared to the usual subregion technique, and the results obtained are more accurate.

The two-dimensionally mapped infinite boundary element (IBE) formulation here used is based on a triangular BE with linear shape functions. One advantage of this formulation over quadratic or higher order elements is that no additional degrees of freedom are added to the original BE mesh by the presence of the IBEs. Thus, the IBEs allow the mesh to be reduced without compromising the accuracy of the result.

The use of IBEs improves the advantages of the alternative multi-region technique, contributing for the low computational cost and allowing a considerable mesh reduction. Furthermore, the results show good agreement with the ones given in other work, confirming the accuracy of the presented formulation.

Keywords: infinite boundary elements, alternative multi-region technique, threedimensional soil, static analysis.
\end{abstract}




\section{Introduction}

Considering specifically infinite multi-domain models, many options are available in the literature and each one of them implies on advantages and disadvantages. However, depending on the problem to be solved, one technique may become more attractive then the others.

Most cases, a numerical approach may be employed. The finite element method (FEM) is still popular (Karakus et al. [1]), however has some disadvantages when compared to other options such as the boundary element method (BEM). The FEM requires the discretization of the infinite domain, implying on a high number of elements and leading to a large and sometimes impracticable processing time. To reduce these inconveniences, some authors use infinite elements together with finite elements, such as Sadecka [2].

It becomes more viable to solve these problems with the BEM, once only the boundary of the domains requires discretization. This allows reducing the problem dimension, implying on less processing time. This advantage is explored in several works (Almeida and Paiva [3]) and more developments are making the BEM even more attractive to future applications.

The classical way to consider domains in contact with the BEM, which is described in details by Brebbia and Dominguez [4], is based on imposing equilibrium and compatibility conditions for all interface points between every pair of domains in contact. These impositions may cause inaccuracies, and numerous blocks of zeros are generated at the final system of equations.

However, Ribeiro and Paiva [5] present an alternative multi-region BEM technique, which does not require equilibrium nor compatibility conditions along the interfaces. Considering a constant Poisson ratio, it is possible to establish relations between the displacement fundamental solutions and to analyze all subdomains as a single solid. Thus, a better continuity between domains in contact is guaranteed and therefore the result accuracy is improved. In addition to that, no blocks of zeros are present at the final system of equations, which is reduced. Thus, better results are obtained in less processing time.

Another way to improve the BEM performance is by using infinite boundary elements (IBEs). The first reference to an IBE was Kagawa et al. [6], in which the shape functions of an origin BE are multiplied by special decay functions. Another type of IBE may be obtained by using mapped functions to relate the local system of coordinates to the global one, as originally shown by Beer and Watson [7]. In those studies that make use of two-dimensional IBEs, such as performed by Moser et al. [8], it may be noted that they are generally based on quadrilateral BEs. An alternative to these type of IBE is given by Ribeiro and Paiva [9], which presented a mapped IBE based on a triangular BE with linear shape functions. One advantage of this approach over quadratic or higher order elements is that no additional degrees of freedom are added to the original $\mathrm{BE}$ mesh by the presence of the IBEs.

The aim of this work is to combine the two BE techniques presented in [5, 9], obtaining a new and more efficient multi-region BE formulation for layered soil 
simulation. The soil is modeled with variable elasticity module and a constant Poisson ratio, as described by Gibson [10]. The results obtained are consistent with those of another author, confirming the accuracy of the presented approach. In addition to that, the use of IBEs contributed for the low computational cost, allowing a considerable mesh reduction.

\section{Boundary element formulation}

The equilibrium of a solid body can be represented by a boundary integral equation called the Somigliana Identity, which for homogeneous, isotropic and linear-elastic domains is

$$
c_{i j}(y) u_{j}(y)+\int_{\Gamma} p_{i j}^{*}(x, y) u_{j}(x) d \Gamma(x)=\int_{\Gamma} u_{i j}^{*}(x, y) p_{j}(x) d \Gamma(x)
$$

Eqn (1) is written for a source point $y$ at the boundary, where the displacement is $u_{j}(y)$. The constant $c_{i j}$ depends on the Poisson ratio and the boundary geometry at $y$. The field point $x$ goes through the whole boundary $\Gamma$, where displacements are $u_{j}(x)$ and tractions are $p_{j}(x)$. The integral kernels $u_{i j}^{*}(x, y)$ and $p_{i j}^{*}(x, y)$ are Kelvin three-dimensional fundamental solutions for displacements and tractions, respectively. Kernel $u_{i j}^{*}(x, y)$ has order $1 / r$ and kernel $p_{i j}^{*}(x, y)$ order $1 / r^{2}$, where $r=|x-y|$, so the integrals have singularity problems when $x$ approaches $y$. Therefore the stronger singular integral, over the traction kernel, has to be defined in terms of a Cauchy Principal Value (CPV).

To solve eqn (1) numerically, the boundary is divided into subregions within which displacements and tractions are approximated by known shape functions. Here these subregions are of two types, finite boundary elements (BEs) and infinite boundary elements (IBEs). The BEs employed are triangular, as shown in fig. 1 with the local system of coordinates, $\xi_{1} \xi_{2}$, and the local node numbering. The

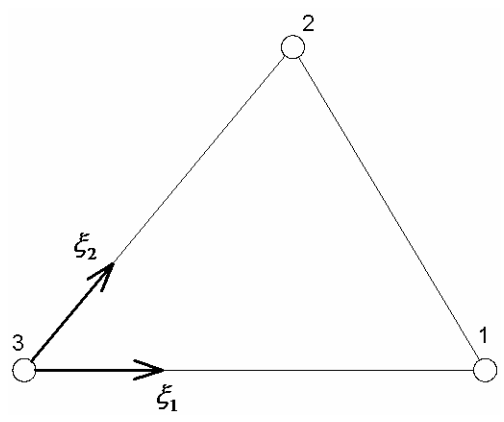

Figure 1: Triangular boundary element. 
following approximations are used for this BE:

$$
u_{j}=\sum_{k=1}^{3} N^{k} u_{j}^{k}, \quad p_{j}=\sum_{k=1}^{3} N^{k} p_{j}^{k}
$$

Eqn (2) relate the boundary values $u_{j}$ and $p_{j}$ to the nodal values of the BE. The BEs have 3 nodes and for each node there are three components of displacement $u_{j}^{k}$ and traction $p_{j}^{k}$. The shape functions $N^{k}$ used for these approximations are

$$
N^{1}=\xi_{1}, \quad N^{2}=\xi_{2}, \quad N^{3}=1-\xi_{1}-\xi_{2}
$$

The same shape functions are used to approximate the boundary geometry:

$$
x_{j}=\sum_{k=1}^{3} N^{k} x_{j}^{k}
$$

where $x_{j}^{k}$ are the node coordinates. The same functions are also used to interpolate displacements and tractions for the IBEs:

$$
u_{j}=\sum_{k=1}^{N p} N^{k} u_{j}^{k}, \quad p_{j}=\sum_{k=1}^{N p} N^{k} p_{j}^{k}
$$

Each IBE has $N p$ nodes and not the 3 that the BEs have. The IBE geometry, on the other hand, is approximated by special mapping functions, as discussed in more detail in Section 3.

By substituting eqns (2) and (5) in eqn (1), eqn (6) is obtained:

$$
\begin{gathered}
c_{i j}(y) u_{j}(y)+\sum_{e=1}^{N_{B E}}\left\{\sum_{k=1}^{3}\left[\Delta p_{i j}^{e k} u_{j}^{k}\right]\right\}+\sum_{e=1}^{N_{I B E}}\left\{\sum_{k=1}^{N p}\left[\Delta^{\infty} p_{i j}^{e k} u_{j}^{k}\right]\right\}= \\
\sum_{e=1}^{N_{B E}}\left\{\sum_{k=1}^{3}\left[\Delta u_{i j}^{e k} p_{j}^{k}\right]\right\}+\sum_{e=1}^{N_{I B E}}\left\{\sum_{k=1}^{N p}\left[\Delta^{\infty} u_{i j}^{e k} p_{j}^{k}\right]\right\}
\end{gathered}
$$

$N_{B E}$ is the number of BEs and $N_{I B E}$ is the number of IBEs. For BEs:

$$
\Delta p_{i j}^{e k}=\int_{\gamma_{e}}|J| N^{k} p_{i j}^{*}(x, y) d \gamma_{e}, \quad \Delta u_{i j}^{e k}=\int_{\gamma_{e}}|J| N^{k} u_{i j}^{*}(x, y) d \gamma_{e}
$$

In eqn (7), $\gamma_{e}$ represents the domain of element $e$ in the local coordinate system and the global system of coordinates is transformed to the local one by the Jacobian $|J|=2 A$, where $A$ is the element area in the global system. On the other hand, for IBEs:

$$
\Delta^{\infty} p_{i j}^{e k}=\left.\int_{\gamma_{e}}\right|^{\infty} J\left|N^{k} p_{i j}^{*}(x, y) d \gamma_{e}, \quad \Delta^{\infty} u_{i j}^{e k}=\int_{\gamma_{e}}\right|^{\infty} J \mid N^{k} u_{i j}^{*}(x, y) d \gamma_{e}
$$

Eqn (8) is analogous to eqn (7), and the calculation of Jacobian $\left|{ }^{\infty} J\right|$ is discussed in Section 3. Integrals of eqns (7) and (8) are calculated by standard 
BEM techniques. Non-singular integrals are evaluated numerically by using integration points. The singular ones, on the other hand, are evaluated by the technique presented in reference [11]. Finally, the free term $c_{i j}$ may be obtained by rigid body motions.

Writing eqn (6) for all boundary nodes leads to the following system:

$$
\Delta p \cdot u=\Delta u \cdot p
$$

The $\Delta p_{i j}^{e k}$ and $\Delta^{\infty} p_{i j}^{e k}$ element contributions, including the free term $c_{i j}$, are assembled into matrix $\Delta p$, while $\Delta u_{i j}^{e k}$ and $\Delta^{\infty} u_{i j}^{e k}$ contributions are assembled into matrix $\Delta u$. Vectors $u$ and $p$ contain all boundary displacements and tractions, respectively. Reorganizing this system so as to separate the known boundary values from the unknown yields a system of equations whose solution is all the unknown boundary values.

\section{Infinite boundary elements}

Three types of mapping are considered, as illustrated in fig. 2 .

a)

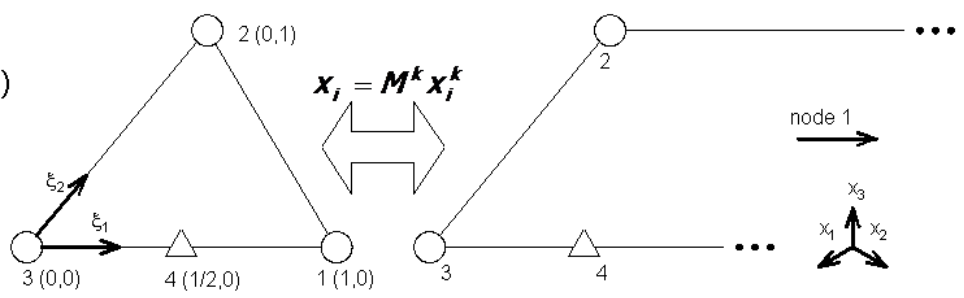

b)

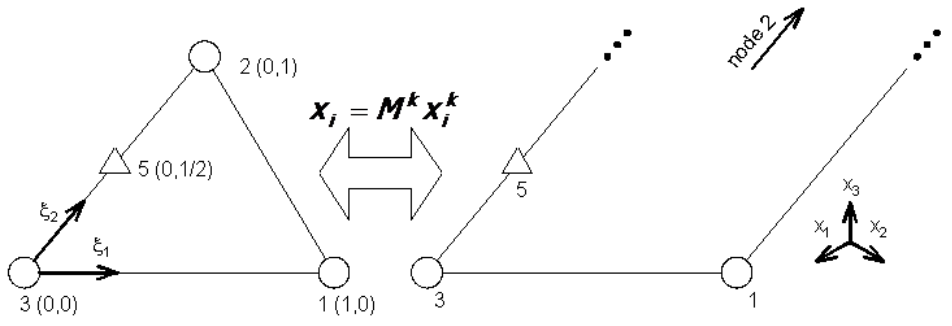

c)

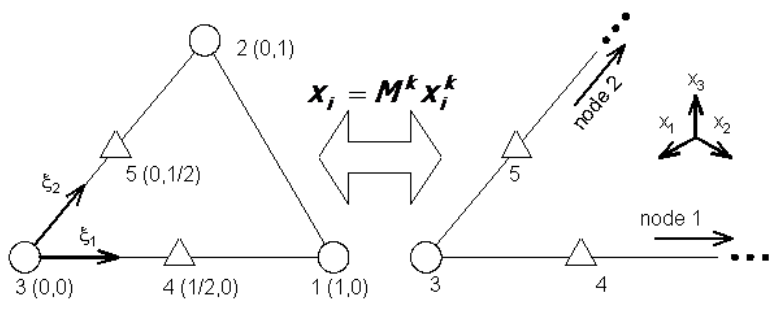

Figure 2: Types of mapping. 
In the first type, represented in fig. $2 \mathrm{a}$, only direction $\xi_{1}$ is mapped and node 1 is placed at infinity. The IBE is represented in the local coordinate system on the left and in the global coordinate system on the right. The global coordinates $x_{i}$ are related to the local ones by special mapping functions, $M^{k}$, and the nodal global coordinates, $x_{i}^{k}$. Node 4 is created only to replace node 1 in the mapping and does not contribute to the integrals.

Fig. $2 \mathrm{~b}$ is analogous to fig. $2 \mathrm{a}$, but in this case only direction $\xi_{2}$ is mapped and node 2 is placed at infinity. Therefore, node 5 is created to facilitate the mapping. Finally, in fig. 2c both local directions are mapped and nodes 1 and 2 are placed at infinity. As a result, the auxiliary nodes 4 and 5 must be created to replace them in the mapping.

Ribeiro and Paiva [9] use auxiliary coordinates $\bar{\xi}_{1}$ and $\bar{\xi}_{2}$ to obtain the mapping functions for each case. When only direction $\xi_{1}$ is mapped, the result is:

$$
\begin{gathered}
M_{1 \infty}^{4}=\bar{\xi}_{1}\left(\xi_{1}\right)=\frac{\xi_{1}}{1-\xi_{1}} \\
M_{1 \infty}^{2}=\xi_{2} \\
M_{1 \infty}^{3}=1-\bar{\xi}_{1}\left(\xi_{1}\right)-\xi_{2}=1-\frac{\xi_{1}}{1-\xi_{1}}-\xi_{2}
\end{gathered}
$$

The symbol " $1 \infty$ " is used to indicate that these expressions are valid if only direction $\xi_{1}$ is mapped. These functions are then employed to relate the local system of coordinates to the global one. In other words:

$$
x_{i}=M_{1 \infty}^{4} x_{i}^{4}+M_{1 \infty}^{2} x_{i}^{2}+M_{1 \infty}^{3} x_{i}^{3}
$$

After obtaining eqn (13), the Jacobian used when only direction $\xi_{1}$ is mapped may be calculated as follows:

$$
\left.\right|^{\infty} J_{1} \mid=\frac{\partial x_{1}}{\partial \xi_{1}} \frac{\partial x_{2}}{\partial \xi_{2}}-\frac{\partial x_{2}}{\partial \xi_{1}} \frac{\partial x_{1}}{\partial \xi_{2}}=\frac{2 A_{1}}{\left(1-\xi_{1}\right)^{2}}
$$

where $A_{1}$ is the area of the triangle drawn between nodes 2,3 and 4 in the global system of coordinates.

For mapping only in direction $\xi_{2}$, the functions obtained are:

$$
\begin{gathered}
M_{2 \infty}^{1}=\xi_{1} \\
M_{2 \infty}^{5}=\bar{\xi}_{2}\left(\xi_{2}\right)=\frac{\xi_{2}}{1-\xi_{2}} \\
M_{2 \infty}^{3}=1-\xi_{1}-\bar{\xi}_{2}\left(\xi_{2}\right)=1-\xi_{1}-\frac{\xi_{2}}{1-\xi_{2}}
\end{gathered}
$$

The symbol " $2 \infty$ " is used to indicate that only direction $\xi_{2}$ is mapped. Therefore, the global system is related to the local one as follows:

$$
x_{i}=M_{2 \infty}^{1} x_{i}^{1}+M_{2 \infty}^{5} x_{i}^{5}+M_{2 \infty}^{3} x_{i}^{3}
$$


and the Jacobian is

$$
\left.\right|^{\infty} J_{2} \mid=\frac{2 A_{2}}{\left(1-\xi_{2}\right)^{2}}
$$

where $A_{2}$ refers to the area of the triangle drawn between nodes 1,3 and 5 in the global system of coordinates.

Finally, for mapping in both directions $\xi_{1}$ and $\xi_{2}$, the mapping functions are

$$
\begin{gathered}
M_{\infty}^{4}=\frac{\xi_{1}}{1-\xi_{1}} \\
M_{\infty}^{5}=\frac{\xi_{2}}{1-\xi_{2}} \\
M_{\infty}^{3}=1-\frac{\xi_{1}}{1-\xi_{1}}-\frac{\xi_{2}}{1-\xi_{2}}
\end{gathered}
$$

The symbol " $\infty$ " is used to indicate that both directions are mapped. The local system of coordinates is related to the global one as follows:

$$
x_{i}=M_{\infty}^{4} x_{i}^{4}+M_{\infty}^{5} x_{i}^{5}+M_{\infty}^{3} x_{i}^{3}
$$

and the Jacobian is now

$$
\left.\right|^{\infty} J_{3} \mid=\frac{2 A_{3}}{\left(1-\xi_{1}\right)^{2}\left(1-\xi_{2}\right)^{2}}
$$

where $A_{3}$ is the area of the triangle drawn between nodes 3,4 and 5 in the global system.

\section{Alternative multi-region formulation}

In fig. 3 a problem with two regions and submitted to arbitrary boundary conditions is presented.

The regions have the same Poisson ratio $\nu$ and different elasticity modules, $E_{1}$ for region $\Omega_{1}$ and $E_{2}$ for region $\Omega_{2}$. The boundary $\Gamma_{1}$ of region $\Omega_{1}$ is divided in two parts, $\Gamma_{12}$ and $\bar{\Gamma}_{1}$. The part of $\Gamma_{1}$ which is in contact with region $\Omega_{2}$ is denoted by $\Gamma_{12}$ and the rest of it is named $\bar{\Gamma}_{1}$. Analogously, region $\Omega_{2}$ boundary $\Gamma_{2}$ is divided into $\Gamma_{21}$ for the contact and $\bar{\Gamma}_{2}$ for the free surface. Consequently:

$$
\Gamma_{1}=\bar{\Gamma}_{1}+\Gamma_{12}, \quad \Gamma_{2}=\bar{\Gamma}_{2}+\Gamma_{21}
$$

Kelvin displacement fundamental solutions for regions $\Omega_{1}$ and $\Omega_{2}$ may be written as:

$$
\begin{aligned}
& u_{i j 1}^{*}=\frac{1}{16 \pi \mu_{1}(1-\nu) r}\left[(3 \nu-4) \delta_{i j}+r_{, i} r_{, j}\right] \\
& u_{i j 2}^{*}=\frac{1}{16 \pi \mu_{2}(1-\nu) r}\left[(3 \nu-4) \delta_{i j}+r_{, i} r_{, j}\right]
\end{aligned}
$$




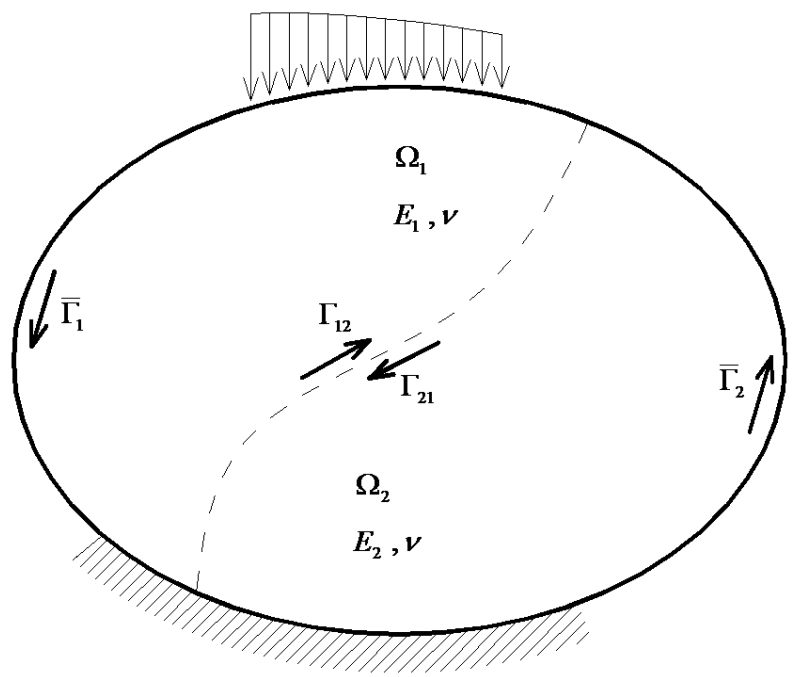

Figure 3: Problem with two regions.

where

$$
\begin{aligned}
& \mu_{1}=\frac{E_{1}}{2(1+\nu)} \\
& \mu_{2}=\frac{E_{2}}{2(1+\nu)}
\end{aligned}
$$

In such a way, the fundamental solutions may be related as follows:

$$
u_{i j 2}^{*}=\frac{E_{1}}{E_{2}} u_{i j 1}^{*}
$$

Eqn (30) may be rewritten as:

$$
u_{i j 2}^{*}=u_{i j 1}^{*}+\frac{\Delta E_{12}}{E_{1}} u_{i j 2}^{*}
$$

where:

$$
\Delta E_{i j}=E_{i}-E_{j}
$$

Kelvin traction fundamental solution may be written as

$$
p_{i j}^{*}=\frac{-1}{8 \pi(1-\nu) r^{2}}\left[\frac{\partial r}{\partial \eta}\left[(1-2 \nu) \delta_{i j}+3 r_{, i} r_{, j}\right]+(1-2 \nu)\left(\eta_{i} r_{, j}-\eta_{j} r_{, i}\right)\right]
$$

One may observe that eqn (33) do not depend on the elasticity module, than it is the same for domains $\Omega_{1}$ and $\Omega_{2}$. Thus, Kelvin traction fundamental solution may be represented as $p_{i j}^{*}$ for both domains. 
The objective is to obtain a single integral equation which considers both regions. In order to do that, the first step is to write the Somigliana Identity integrating only the boundary of region $\Omega_{1}$. Maintaining the same source point, the Somigliana Identity is then again written but now integrating only the boundary of region $\Omega_{2}$. By adding these two expressions, an initial version of the final equation is obtained. The next step is to substitute eqn (31) into this initial version and to develop the expression. This demonstration is detailed by Ribeiro and Paiva [5], and the final result is

$$
\begin{gathered}
{\left[c_{i j 1}+\frac{E_{2}}{E_{1}} c_{i j 2}\right] u_{j}+\int_{\bar{\Gamma}_{1}} p_{i j}^{*} u_{j} d \bar{\Gamma}_{1}+\frac{E_{2}}{E_{1}} \int_{\bar{\Gamma}_{2}} p_{i j}^{*} u_{j} d \bar{\Gamma}_{2}-\frac{\Delta E_{12}}{E_{1}} \int_{\Gamma_{21}} p_{i j}^{*} u_{j} d \Gamma_{21}} \\
=\int_{\bar{\Gamma}_{1}} u_{i j 1}^{*} p_{j} d \bar{\Gamma}_{1}+\int_{\bar{\Gamma}_{2}} u_{i j 1}^{*} p_{j} d \bar{\Gamma}_{2}
\end{gathered}
$$

Variables $x$ and $y$ were omitted in order to reduce the expression. Extending it to an arbitrary number of domains, it becomes:

$$
\begin{gathered}
\left\{\sum_{s=1}^{n d}\left[\frac{E_{s}}{E_{1}} c_{i j s}\right]\right\} u_{j}+\sum_{e=1}^{n e}\left[\frac{E_{e}}{E_{1}} \int_{\bar{\Gamma}_{e}} p_{i j}^{*} u_{j} d \bar{\Gamma}_{e}\right]+\sum_{c=1}^{n c}\left[\frac{\Delta E_{m n}}{E_{1}} \int_{\Gamma_{m n}} p_{i j}^{*} u_{j} d \Gamma_{m n}\right]= \\
=\sum_{e=1}^{n e}\left[\int_{\bar{\Gamma}_{e}} u_{i j 1}^{*} p_{j} d \bar{\Gamma}_{e}\right]
\end{gathered}
$$

In eqn (35), the total number of domains is $n d$, the number of contact boundaries is $n c$ and the number of external boundaries is ne. The first summation represents the coefficient $c_{i j}(y)$ of eqn (1), which contributes in the matrix $\Delta p$ of eqn (9). Before calculating it all coefficients $c_{i j s}$, one for each domain, must be known. More details about this formulation are provided by Ribeiro and Paiva [5].

In Section 2, eqn (1) was used as a starting point to obtain the BEM system of equations which solution is the unknown boundary values. If the same steps are repeated for eqn (35), valid for multi-regions, a similar system of equations is obtained. The unknowns of this new system are the non-prescribed boundary values plus the interface displacements. The total number of unknowns is reduced when compared to the classic multi-region technique described in reference [4], once the interface tractions are not included in this case. This justifies why the alternative formulation leads to less time processing. A better interface continuity is also guaranteed, once all regions are modeled as a single solid.

\section{Example}

This example aims to analyze an infinite non-homogeneous domain problem with the proposed formulation. The domain considered is composed by two layers of different elasticity module, as illustrated in fig. 4.

Layer 1 has a $9000 \mathrm{kN} / \mathrm{m}^{2}$ elasticity module, a 0.5 Poisson ratio and $15 \mathrm{~m}$ of thickness. Layer 2 has a $900 \mathrm{kN} / \mathrm{m}^{2}$ elasticity module, a 0.5 Poisson ratio and 
284 Boundary Elements and Other Mesh Reduction Methods XXXII

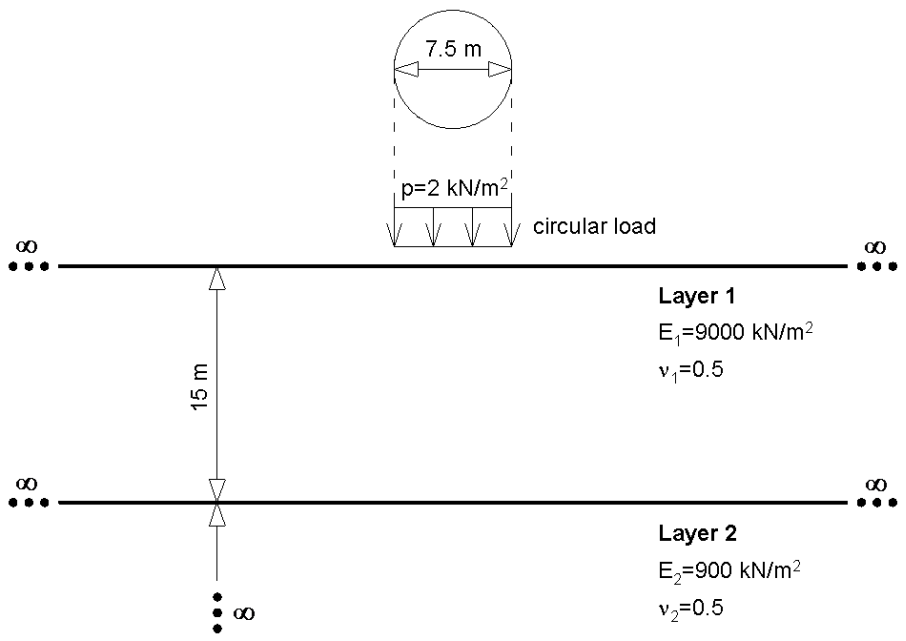

Figure 4: Layered soil problem.

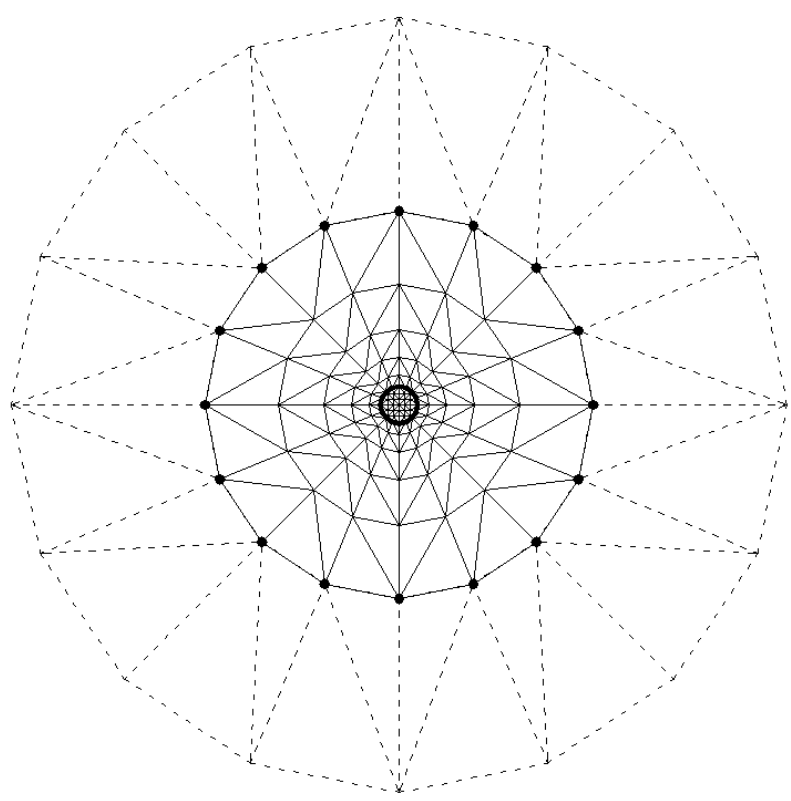

Figure 5: Mesh employed.

infinite thickness. Both layers are infinite in radial directions. A vertical circular 2 $k N / m^{2}$ uniform loading with a $7.5 \mathrm{~m}$ diameter is applied at the top layer surface.

This problem was simulated using two identical meshes, one for the surface and another for the contact between layers, totalizing 242 nodes, 448 BEs and 64 IBEs. The surface mesh may be visualized in fig. 5 , in which the polygon outlined in the 
Table 1: Vertical displacement $\left(m \times 10^{-3}\right)$.

\begin{tabular}{|c|c|c|c|c|}
\hline Number of nodes & No IBEs used & Error (\%) & Mesh with IBEs & Error (\%) \\
\hline \hline Analytical & 2.5000 & & 2.5000 & \\
\hline \hline 242 & 2.0874 & 16.5 & 2.5123 & 0.5 \\
\hline 274 & 2.3865 & 4.5 & 2.5209 & 0.8 \\
\hline 306 & 2.5033 & 0.1 & 2.5212 & 0.8 \\
\hline 338 & 2.5207 & 0.8 & 2.5213 & 0.9 \\
\hline
\end{tabular}

center corresponds to the loaded area, the dashed lines represent the IBEs and the rest of the mesh is composed of BEs. The points marked at the perimeter of the BE mesh receive all the influence of the IBEs.

Using the values adopted in this example and applying the analytical solution given by Burmister [12], a $2.5000 \times 10^{-3} \mathrm{~m}$ vertical displacement is obtained for the central point of the circle. Simulating this problem with the mesh of fig. 5, a vertical displacement of $2.5123 \times 10^{-3} \mathrm{~m}$ was obtained. This value agrees with the analytical solution, with an error of $0.5 \%$. In order to evaluate the influence of the IBEs, the example was simulated with the same BE mesh but without the IBEs. A displacement of $2.0874 \times 10^{-3} \mathrm{~m}$ was then obtained, with the higher error of $16.5 \%$ relative to the analytical value.

In order to improve this precision more BEs and degrees of freedom were added at the mesh limits. In this way, the values in table 1 were obtained.

As may be observed, 306 nodes were needed for the BE mesh to overcome the precision of the 242 node mesh with IBEs. Comparing these two values it may be concluded that, in this example, to maintain the error below $0.5 \%$, the use of IBEs allows a mesh reduction of $21 \%$.

\section{Conclusions}

In this paper, an alternative multi-region $\mathrm{BE}$ technique was combined with a IBE formulation in order to obtain an efficient numerical tool for layered soil simulation. Electing one domain as a reference and establishing relations between its displacement fundamental solution and the ones of the other regions allows integrating all domains as a single solid. This approach eliminates the need of equilibrium and compatibility relations between the different media. Therefore, better results may be obtained in less processing time.

The IBEs employed have the advantage of not increasing the original number of degrees of freedom, as demonstrated in the example presented. The results obtained with the IBEs showed good agreement with an analytical solution, and the use of IBEs promoted a mesh reduction of $21 \%$. 


\section{Acknowledgements}

We wish to thank the Structural Engineering Department of the University of São Paulo and the research council FAPESP.

\section{References}

[1] Karakus, M., Ozsan, A. \& Basarir, H., Finite element analysis for the twin metro tunnel constructed in ankara clay, turkey. Bulletin of Engineering Geology and the Environment, 66, pp. 71-79, 2007.

[2] Sadecka, L., A finite/infinite element analysis of thick plate on a layered foundation. Computers and Structures, 76(5), pp. 603-610, 2000.

[3] Almeida, V.S. \& Paiva, J.B., Static analysis of soil/pile interaction in layered soil by bem/bem coupling. Advances in Engineering Software, 38(11-12), pp. 835-845, 2007.

[4] Brebbia, C.A. \& Dominguez, J., Boundary elements an introductory course. Computational Mechanics Publications: London, 1992.

[5] Ribeiro, D.B. \& Paiva, J.B., An alternative multi-region bem technique for three-dimensional elastic problems. Engineering Analysis with Boundary Elements, 33(4), pp. 499-507, 2009.

[6] Kagawa, Y., Yamabuchi, T. \& Kitagami, S., The infinite boundary element method and its application to a combined finite boundary element technique for unbounded field problems. The international journal for computation and mathematics in electrical and electronic engineering (COMPEL), 2(4), pp. 179-193, 1983.

[7] Beer, G. \& Watson, J.O., Infinite boundary elements. International Journal for Numerical Methods in Engineering, 28(6), pp. 1233-1247, 1989.

[8] Moser, W., Duenser, C. \& Beer, G., Mapped infinite elements for threedimensional multi-region boundary element analysis. International Journal for Numerical Methods in Engineering, 61(3), pp. 317-328, 2004.

[9] Ribeiro, D.B. \& Paiva, J.B., A new infinite boundary element formulation applied to three-dimensional domains. The 2009 International Conference of Mechanical Engineering, International Association of Engineers (IAENG): London, England, 2009.

[10] Gibson, R.E., Some results concerning displacements and stresses in a nonhomogeneous elastic half-space. Géotechnique, 17(1), pp. 58-67, 1967.

[11] Guiggiani, M. \& Gigante, A., A general algorithm for multidimensional cauchy principal value integrals in the boundary element method. Journal of Applied Mechanics, 57, pp. 906-915, 1990.

[12] Burmister, D.M., The general theory of stresses and displacements in layered systems 1. Journal of Applied Physics, 16(2), pp. 89-96, 1945. 